$\underline{\text { Research Articles }}$

\title{
The impact of Syrian refugees on patient demographics and type 2 diabetes across five public health centers in Lebanon
}

\author{
Peter Makhoul ${ }^{1}$ (D), Raya Tcheroyan ${ }^{2}$ (D) ${ }^{\text {a }}$, Walid Ammar ${ }^{3}$, Randa Hamadeh ${ }^{4}$, Pierre Zalloua ${ }^{5}$ (D) Ghassan Nabbout ${ }^{5}$ \\ ${ }^{1}$ Faculty of Medicine, University of Massachusetts Medical School, Worcester, Massachusetts, USA, ${ }^{2}$ Faculty of Medicine, American University of \\ Beirut Medical School, Lebanon, ${ }^{3}$ Global Health Team of Experts, Ministry of Public Health, Beirut, Lebanon, ${ }^{4}$ Faculty of Medicine, Université Saint- \\ Joseph de Beyrouth, Lebanon, 5 Faculty of Medicine, University of Balamand, Lebanon \\ Keywords: lebanon, syrian refugees, diabetes \\ https://doi.org/10.29392/001c.24948
}

Journal of Global Health Reports

Vol. 5, 2021

\begin{abstract}
Background
The war in Syria triggered the influx of a large number of refugees into neighboring countries, particularly Lebanon. In 2019, the Syrian population made up $13.33 \%$ of the total population in Lebanon. This shift in demographics influenced many sectors in the country, including the healthcare system.

The purpose of this study is to analyze the impact of Syrian refugees on the Lebanese healthcare system across five public health centers (PHCs) and the prevalence of type II diabetes (T2D) in these patients.
\end{abstract}

\section{Methods}

Patient data was collected across five PHCs from different regions in Lebanon. Comparisons between Lebanese and Syrian patients were analyzed for demographics, relative number of patients, and prevalence of T2D.

\section{Results}

We found that the number of patients admitted to PHCs has increased over the last few years, in both Syrian and Lebanese populations, putting more strains on an already threatened sector. In addition, the increase in Lebanese patients is significantly higher after 2015, and it parallels the surge in Syrian immigrants. The prevalence of T2D was found to be increasing over the years among the two populations, with a statistically significant increase in the Lebanese population after 2015. However, the prevalence of T2D was found to be statistically lower than the reported national prevalence in both groups, possibly due to inadequate rates of screening.

\section{Conclusions}

There has been an increase in admissions to PHCs as well as rates of T2D in Lebanon and in the Syrian population. Inadequate screening could be the reason behind the lower prevalence of T2D in our study compared to the reported national prevalence.

Since March 2011, the war in Syria has forced a massive number of Syrians to seek refuge in neighboring countries, such as Lebanon, Jordan, and Turkey. At the beginning of 2015 , the number of Syrian refugees reached the million mark, with approximately $1,120,000$ registered according to the United Nations High Commissioner for Refugees (UNHCR). ${ }^{1}$ Today, Lebanon remains the country with the highest number of Syrian refugees in the world, with a total number of 910,256 registered refugees comprising approximately $13.33 \%$ of the total population. ${ }^{2}$

Unlike most other countries, the Lebanese government has resisted setting up refugee camps, citing the fear of these refugees staying permanently in the country. ${ }^{3}$ This forced the majority of these refugees to find their own homes or congregate in very small apartments and rooms, often in areas that were already congested with Lebanese citizens. Approximately $18 \%$ of these refugees live in makeshift tents that they set up themselves or in collaboration with the UNHCR. ${ }^{4}$ This unprecedented crisis has thus naturally had a huge impact on both the Lebanese and Syrian populations residing in Lebanon.

Lebanon itself has also suffered from an unstable politi- 
cal climate since the beginning of the civil war in 1975. Following almost two decades of war, the country's economy as well as its healthcare system have struggled with continuous instability, putting at risk the resilience and sustainability of their services to its population..$^{5}$ The Syrian refugee crisis has created additional obstacles to the Lebanese healthcare sector, overwhelming it with the massive number of refugees. ${ }^{6}$ The refugees' demographics also vary from those of the Lebanese, as the former has a higher proportion of younger inhabitants than the latter. The UNHCR estimates that Syrian refugees aged 0-4 years amount to $15.2 \%$ of their population, while Lebanese population statistics estimate that this age group accounts for around $7.8 \%$ of the Lebanese. ${ }^{7}$ Similarly, the proportion of Syrian refugees aged 5-11 has been estimated at $24.8 \%$, while that of Lebanese aged 5-14 years is around 16.2\%. Most of these refugees reside in the Bekaa region of Lebanon (37.8\%), followed by North Lebanon (26.5\%), Beirut (24.5\%), and South Lebanon (11.2\%). ${ }^{1,7}$ This is in contrast to the spread of the Lebanese population, which primarily resides in Beirut (28.1\%) and less in the Bekaa region (14.65\%). ${ }^{8}$ These differences in demographics provide an added layer of complexity to the capacity of Lebanese resources to adequately provide for refugees.

Because of these added challenges, Primary Healthcare Centers (PHCs) in Lebanon have made it their mission to accommodate many of the displaced Syrians in addition to their usual cohort of Lebanese patients. The Lebanese Ministry of Public Health has been working with 238 public PHCs as of 2019, with increasing quality of service over the years. ${ }^{9}$ With the help of the UNHCR, Lebanon has been able to provide the Syrian refugees with basic healthcare services; for example, the UNHCR offers unregistered Syrian women one visit to a PHC along with various other services. ${ }^{6}$ However, the rudimentary living conditions of the refugees and the uncertainties associated with living in in a foreign country without a prospect of a near return to the home country can be very challenging.

Studies have consistently shown that refugees endure high levels of acute stress following their forced displacement. ${ }^{10}$ Depriving refugees from accessing treatment for chronic non-communicable diseases (NCDs) has been identified as a condition threatening their survival and quality of life. ${ }^{11}$ Health services have, however, mainly focused on the detection of communicable disease among refugees, and data is still limited on chronic NCDs, particularly type 2 diabetes (T2D), despite the strong evidence linking stress and lifestyle changes with diabetes on the individual level. ${ }^{12,13}$ Novak et al ${ }^{14}$ showed that even when controlling for age, socioeconomic status (SES), and other typical T2D risk factors, self-perceived permanent stress remained a key long-term predictor of the development of this disease in their patient population.

Studies conducted on Syrian refugees in the U.S., Lebanon and neighboring countries like Jordan have unanimously shown a high prevalence of diabetes, with more than half of adult refugees suffering from T2D or at least one other metabolic syndrome disease component; this is compounded by a lack of feasible access to healthcare. ${ }^{15-17}$ The relationship between abrupt displacement and lifestyle changes with rates of T2D among both refugees and their

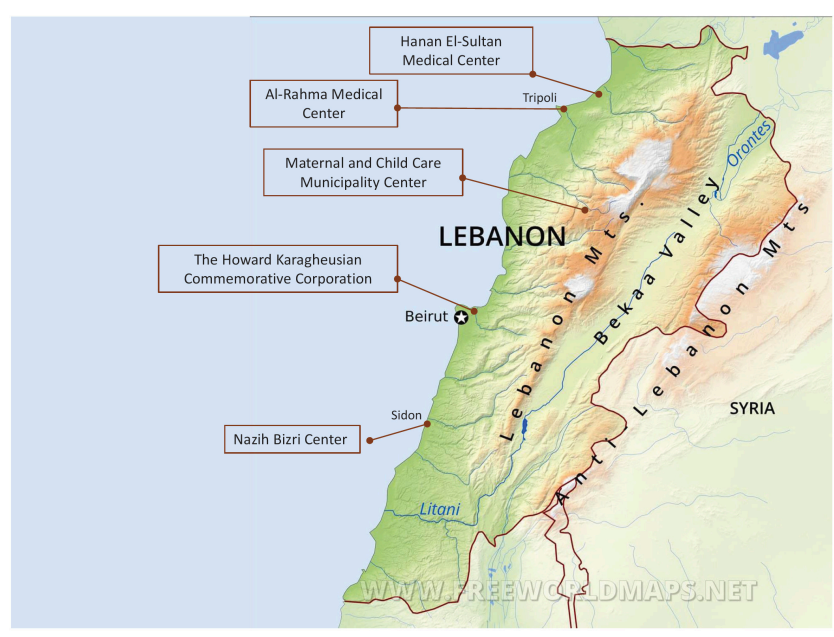

Figure 1. Geographical distribution of the primary healthcare centers across Lebanon.

host population is thus a topic that warrants extensive study.

In this study, we focused on analyzing the demographic characteristics of the Syrian refugees as compared to the Lebanese population, and the impact that the refugees' influx had on the Lebanese health system across five different public health centers. We analyzed the prevalence of T2D and its evolution over the years among both Lebanese and Syrian patients, in an attempt to find a correlation between diabetes and stress within these populations, taking the status as refugees and the worsening Lebanese economic situation as major stress contributors.

\section{METHODS}

Patient data were collected from five PHCs across Lebanon between 2010 and 2019 inclusive to allow for a longitudinal study design. Our data was obtained from multiple sources, primarily the Lebanese Ministry of Public Health database and data from the designated centers.

The total population studied consisted of a total of 131,016 self-admitted patients with information about the date of their admission, sex, age, nationality, height, weight, social history, and past-medical history. The study was approved by the IRB of the University of Balamand.

The centers selected for the study are located in five different regions across Lebanon (Fig 1): Dr. Nazih Bizri center in Saida, Hanan El-Sultan Medical Center in Miniyeh, Al-Rahma Medical Center in Tripoli, Maternal and Child Care Municipality Center in Meshmesh and The Howard Karagheusian Commemorative Corporation in Bourj Hammoud. These five centers were chosen because of their good geographical spread from the North to the South of Lebanon, thus allowing for a good representation of the general population. ${ }^{18}$ The centers also stored their data electronically, making the retrieval of previous years' data possible.

We first analyzed the number of Lebanese and Syrian patients visiting each center between 2010 and 2019 and investigated the impact of the number of Syrian refugees into 
Lebanon on the numbers of Lebanese patients visiting these same PHCs. We also compared the numbers across centers to see if differences could be attributed to regional disparities in the distribution of refugees in Lebanon.

Patients of all ages were considered for this section. In a follow up analysis, only 20-year-old patients or older were taken into account to comment on the prevalence of T2D in both Lebanese and Syrian populations and to investigate whether stress and life-style changes that are associated with being a refugee increase that prevalence.

\section{RESULTS}

We recorded a steady increase in the total number of both Syrian and Lebanese patients across all centers, from 4,898 (1608 Lebanese males, 2823 Lebanese females, 226 Syrian males and 241 Syrian females) in 2014 to 13,873 in 2015, peaking at 27,966 in 2018. The data for 2019 reflects midyear values, and further increases that year compared to the previous ones remain possible (Fig. 2). In 2015 in particular, there was a considerable surge in the number of Syrian patients seeking care at the PHCs, from 241 females and 226 males in 2014 to 2,765 females and 1,666 males in 2015, representing a $948 \%$ increase in the total share of Syrian patients. This increase continued into 2016, with 6,697 Syrian females and 4,391 males presenting for care, and numbers have been persistently high since then. This was also accompanied by a steady increase in the number of Lebanese patients with the highest increase occurring in 2015.

The influx of Syrian refugees gradually decreased or even reversed the ratio of Lebanese/Syrian patients across all centers and this is due to the steady increase in the number of Syrian refugees.

The Maternal Child Care Municipality Center, Hanan ElSultan Medical Center and Al Rahma Medical Center maintained a constant Lebanese to Syrian ratio above 1, welcoming more Lebanese patients than Syrian patients over the years. However, Al Rahma Medical Center has been receiving a higher number of Syrian patients from 2015 onwards with a ratio getting closer to 1 (from 349.3 in 2013 to 5.8 in 2019).

Prior to the start of the war in Syria, the ratio of Lebanese to Syrian patients at Dr. Nazih Bizri Center was 316.81 in 2010, reflecting a massive predominance of Lebanese patients. However, starting 2011 and the onset of the war, the influx of Syrian refugees led to a decrease in this ratio, from 223.00 in 2011 to 0.69 in 2019 , reflecting a shift in this patient population from being predominantly Lebanese to predominantly Syrian. Finally, Howard Karagheusian Commemorative Center is the only PHC that has constantly taken in more Syrian patients, with a ratio of Lebanese to Syrian patients less than 1, starting at 0.01 in 2015 and increasing to 0.63 in 2019 (Fig.3).

Across centers, Al-Rahma Medical Center had the largest total number of patients, with 35,738 patients presenting between the years 2010 and 2019. The Howard Karaghuesian Commemorative Corporation admitted a higher yearly average of patients, with an average of 6,316 patients a year. All PHCs had more Lebanese than Syrian patients, with the

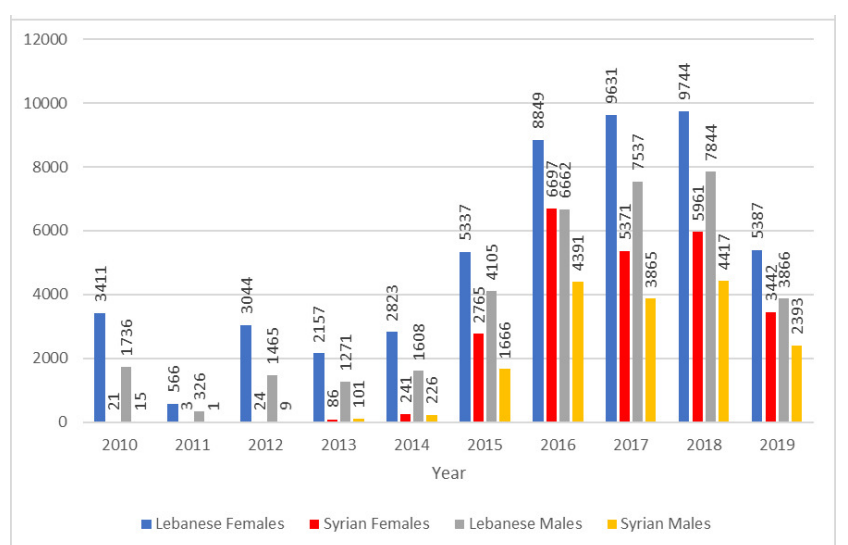

Figure 2. Total number of Lebanese and Syrian patients across all centers.

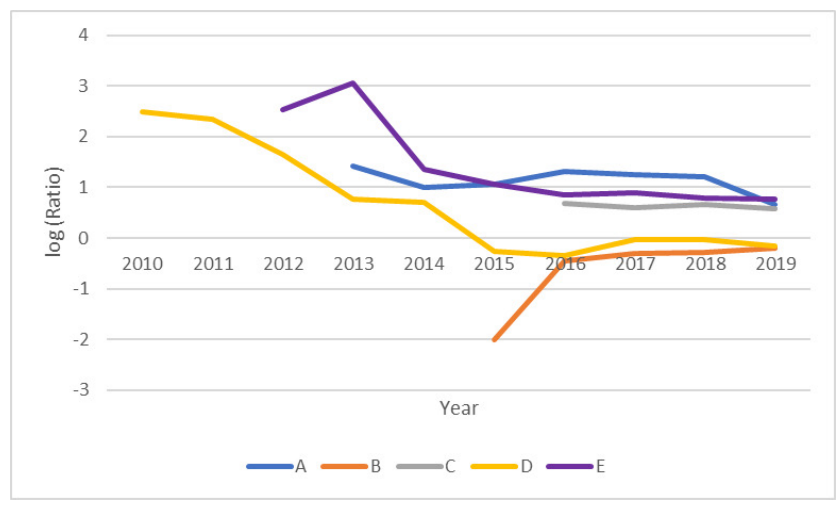

Figure 3. The ratio of Lebanese to Syrian patients across the five primary healthcare centers using a log scale.

In-text values are reported as original values. A: Maternal and Child Care Munic ipality Center; B: Howard Karagheusian Commemorative Center ; C: Hanan ElSultan Medical Center; D: Dr. Nazih Bizri Center; E: Al-Rahma Medical Center.

exception of the Howard Karagheusian Center, which reported 8,914 male and 12,305 female (21,219 total) Syrian patients compared to 3,658 male and 5,449 female $(9,107$ total) Lebanese patients in the last 5 years (Table 1). The largest number of patients with T2D was in the Howard Karagheusian Center, with 1,421 cases reported in the last 5 years, compared to 254 , the lowest number of diabetics, in the Maternal and Child Care Municipality Center over the last 7 years (Table. 1$)$.

Female patients made up the majority of patients in both Lebanese and Syrian populations across four of the five PHCs with a significant difference in percentages between the different sexes in both patients aged 20 to 29 and 30 to 49 (63\% to $37 \%$ in the Lebanese population and $81 \%$ to $19 \%$ in the Syrian population) (Table 2).

The majority of Lebanese patients coming to the centers were either young adults aged 30 to 49 or young children aged 0 to 9 . In the Syrian population, almost half the patients are children aged 0 to 9 (44\%) compared to only $22 \%$ of Lebanese patients in that age range (Table 2). 
Table 1. Demographic data across the primary healthcare centers.

\begin{tabular}{|c|c|c|c|c|c|c|c|c|c|}
\hline \multirow[t]{2}{*}{ Center } & \multirow{2}{*}{$\begin{array}{c}\text { Total } \\
\text { patients }\end{array}$} & \multirow{2}{*}{$\begin{array}{l}\text { New admissions per } \\
\text { year }\end{array}$} & \multicolumn{2}{|c|}{ Lebanese patients } & \multicolumn{2}{|c|}{ Syrian patients } & \multirow[t]{2}{*}{ Others } & \multirow{2}{*}{$\begin{array}{l}\text { Mean } \\
\text { age }\end{array}$} & \multirow{2}{*}{$\begin{array}{l}\text { Patients with } \\
\text { T2D }\end{array}$} \\
\hline & & & Male & Female & Male & Female & & & \\
\hline $\begin{array}{c}\text { The Maternal and Child Care Municipality } \\
\text { Center }\end{array}$ & 15192 & 2170 & 6521 & 7,503 & 557 & 546 & 65 & 27.4 & 254 \\
\hline Hanan El-Sultan Medical Center & 17826 & 3565 & 6257 & 8120 & 1452 & 1862 & 135 & 23.9 & 287 \\
\hline Dr. Nazih Bizri center & 30739 & 3074 & 6645 & 10857 & 4767 & 7797 & 673 & 31.1 & 857 \\
\hline Al-Rahma Medical Center & 35738 & 4467 & 13139 & 19020 & 1394 & 2101 & 84 & 25.8 & 283 \\
\hline $\begin{array}{c}\text { The Howard Karagheusian Commemorative } \\
\text { Center }\end{array}$ & 31582 & 6316 & 3658 & 5449 & 8914 & 12305 & 1256 & 20.8 & 1421 \\
\hline
\end{tabular}


Table 2. Proportion of males and females of Lebanese and Syrian patients by age group.

\begin{tabular}{|c|c|c|c|c|c|c|c|}
\hline \multirow[b]{2}{*}{ Age (years) } & \multicolumn{3}{|c|}{ Lebanese } & \multicolumn{3}{|c|}{ Syrian } & \multirow[b]{2}{*}{ P-value } \\
\hline & $\begin{array}{l}\text { Population } \\
\mathrm{N}=87189\end{array}$ & Male & Female & $\begin{array}{l}\text { Population } \\
N=41728\end{array}$ & Male & Female & \\
\hline 0 to 9 & 19416 & $51 \%$ & $49 \%$ & 18211 & $52 \%$ & $48 \%$ & $<0.001$ \\
\hline 10 to 19 & 16170 & $48 \%$ & $52 \%$ & 7023 & $46 \%$ & $54 \%$ & $<0.001$ \\
\hline 20 to 29 & 14828 & $37 \%$ & $63 \%$ & 5684 & $19 \%$ & $81 \%$ & $<0.001$ \\
\hline 30 to 49 & 19908 & $33 \%$ & $67 \%$ & 7477 & $28 \%$ & $72 \%$ & $<0.001$ \\
\hline $50+$ & 16867 & $39 \%$ & $61 \%$ & 3333 & $38 \%$ & $62 \%$ & $<0.001$ \\
\hline
\end{tabular}

Data on the prevalence of T2D among all patients aged between 20 and 79 years were collected and analyzed via a chi-squared test. Among Lebanese patients, a progressive increase in rates of T2D from $1.57 \%$ in 2010 to $7.62 \%$ in 2019 was shown. The increase in prevalence was observed in both males and females (Table 3). However, this prevalence was lower than what is reported in the literature for the Lebanese population as a whole, $7.8 \%$ in 2010 with a steady annual increase reaching $11.2 \%$ in $2019.19,20$

The change in prevalence of T2D among Syrian refugees aged between 20 and 79 years was also calculated, showing a progressive increase in this prevalence from 2015 onwards (Table 3). This was, however, still lower than the national rate of T2D in Syria. ${ }^{21,22}$ Data from years prior to 2015 were not used because of the small number of Syrian patients presenting to the PHCs in those years (often less than 100 patients per year).

\section{DISCUSSION}

The uneven distribution of patients in the PHCs parallels the distribution of the Lebanese population and the number of refugees in each region. The continuous influx of refugees after 2015 increased the number of Syrian patients which was balanced by a variable increased demand for PHCs by Lebanese due to the worsening economic situation that affected the whole Lebanese economy with some local regional variances. Medical care at these PHCs is heavily subsidized by the Lebanese government and many charitable and non-governmental organizations.

For example, Mechmech is a region in the Jbeil district, which has a characteristically low number of refugees, estimated between 2753 and 7646 individuals in $2016 .{ }^{23}$ This could explain the small number of Syrian patients compared to Lebanese at the Maternal Child Care Municipality Center.

In contrast, the Howard Karagheusian Commemorative Corporation, is located in Bourj Hammoud; the predominance of Syrian patients at this center is explained by the large population of refugees in Bourj Hammoud. According to an article by the UN Habitat (2017), it was reported that a district in Bourj Hammoud had 14,760 residents in Nov. 2016, of whom two thirds were Syrian refugees.

The increasing poverty rates in Lebanon as well as fur-

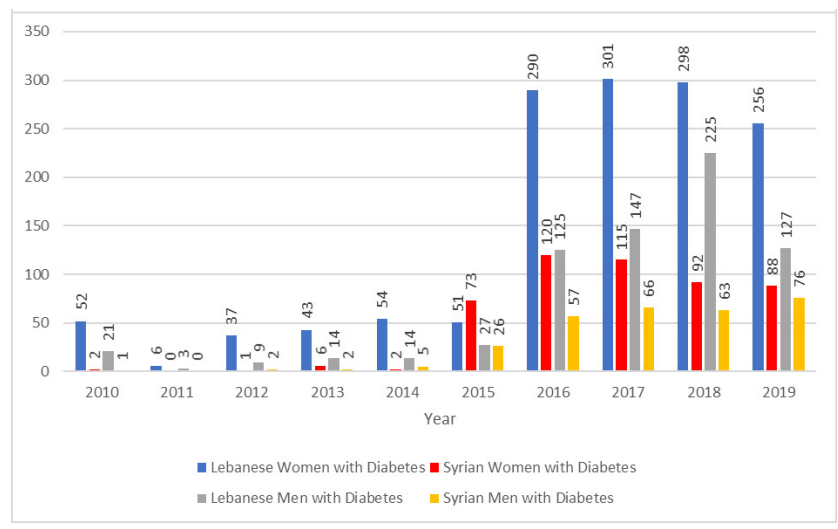

Figure 4. Total Number of Lebanese and Syrian patients with diabetes across all primary healthcare centers.

ther decline in its economic state may have collectively had huge impacts on both Syrian and Lebanese families' abilities to afford care at more expensive and specialized healthcare centers. This might explain the exponential increase in the total number of patients across all PHCs. According to the World Bank, ${ }^{24}$ the start of the Syrian crisis in 2011 was accompanied by a simultaneous increase in Lebanese families living at or below the poverty threshold. In addition to the already existing 1 million Lebanese families living in poverty, an additional 200,000 now met these criteria. According to Doocy et al. ${ }^{25}$ more than half of the Lebanese population lacks proper insurance plans, making PHCs a more accessible option for seeking care. The increase in demand for PHCs by both Lebanese and Syrian patients can thus be attributed to both limited available resources and progressively worsening economic capabilities. Furthermore, for Syrian patients in particular, some PHCs have put forth agreements to welcome and treat Syrian refugees, such as the agreement signed between the Dr. Nazih Bizri Center and the International Medical Corps in 2015. ${ }^{1,26}$ These efforts to attract and tailor care towards this population could provide further justification for their increasing numbers at these centers. This is highlighted in Figure 2, in which the total number of Syrian patients at this center increased from 187 in 2014 to 1,951 in 2015. 
Table 3. Prevalence of T2D among Lebanese population aged between 20 and 79 years, admitted to the pimary health centers.

\begin{tabular}{|c|c|c|c|c|c|c|c|c|c|c|c|c|}
\hline Country & Sex & 2010 & 2011 & 2012 & 2013 & 2014 & 2015 & 2016 & 2017 & 2018 & 2019 & $P$-value \\
\hline \multirow[t]{3}{*}{ Lebanon } & Female & $1.64 \%$ & $1.24 \%$ & $1.54 \%$ & $2.65 \%$ & $2.32 \%$ & $1.43 \%$ & $5.16 \%$ & $5.00 \%$ & $5.42 \%$ & $7.56 \%$ & $<0.001$ \\
\hline & Male & $1.43 \%$ & $1.30 \%$ & $1.33 \%$ & $2.04 \%$ & $1.45 \%$ & $1.18 \%$ & $3.28 \%$ & $3.77 \%$ & $6.46 \%$ & $7.74 \%$ & $<0.001$ \\
\hline & Total & $1.57 \%$ & $1.26 \%$ & $1.49 \%$ & $2.47 \%$ & $2.06 \%$ & $1.33 \%$ & $4.65 \%$ & $4.51 \%$ & $5.82 \%$ & $7.62 \%$ & $<0.001$ \\
\hline \multirow[t]{3}{*}{ Syria } & Female & - & - & - & - & - & $4.53 \%$ & $3.58 \%$ & $4.22 \%$ & $3.69 \%$ & $4.95 \%$ & 0.008 \\
\hline & Male & - & - & - & - & - & $5.09 \%$ & $5.58 \%$ & $6.35 \%$ & $6.65 \%$ & $9.75 \%$ & - \\
\hline & Total & - & - & - & - & - & $4.66 \%$ & $4.04 \%$ & $4.79 \%$ & $4.50 \%$ & $6.41 \%$ & - \\
\hline
\end{tabular}


Another important finding is that in both the Syrian and Lebanese population, females represent the majority of patients visiting the center, especially women of childbearing age. One potential explanation is that within these particular age groups, women tend to be mothers who accompany their children to these centers to a larger extent than their fathers do. They thus schedule visits for themselves and their children simultaneously. In a study targeting Lebanese and Syrian women of reproductive age conducted by Truppa et $\mathrm{al}^{27}$ it was found that the majority of women visiting PHCs were mainly seeking care for their children, with $37.8 \%$ of women reporting this as their primary reason for presenting.

Finally, it was also noted that the majority of Syrian patients are young children aged 0 to 9 . PHCs provide vaccinations to children at a lower cost than private medical practices, which makes these centers very attractive to families that struggle to meet higher costs. ${ }^{5}$

Across PHCs, the prevalence of T2D was shown to increase steadily over the years. A potential explanation could be the stress associated with the socio-economic and psychological crises that refugees face. The potential association between stress and T2D has indeed been previously studied. According to Kyrou and Tsigos, ${ }^{28}$ chronic stress and continued activation of the sympathetic nervous system increase the risk of developing obesity, metabolic syndrome, and T2D. Mooy et $\mathrm{al}^{29}$ determined that chronic psychological stress resulting from major life events was associated with an increased prevalence of T2D. This relationship was also studied by Chrousos, ${ }^{30}$ who showed that chronic stress as well as poor socioeconomic status, both of which Syrian refugees suffer from, can lead to chronic diseases like T2D. A population-based study by Connolloy et $\mathrm{al}^{31}$ illustrated that an inversely proportional relationship exists between socioeconomic status and the prevalence of T2D, especially in underprivileged areas. They attributed this correlation to multiple possible factors, including low birth weight, inactivity, and limited routine glucose blood tests. Studies in Lebanon showed a significant prevalence of depression among patients suffering from T2D. ${ }^{19}$ All these factors are present among the Syrian and Lebanese populations, who, as a result of the psychological and socioeconomic impacts of the war as well as the limited opportunities and resources available in Lebanon, could ultimately suffer from increased rates of long-term, chronic diseases like T2D.

Within the Lebanese population itself, we also found that the prevalence of T2D was statistically higher from 2015 onwards compared to previous years. This coincides with the timing of the surge of Syrian refugees into Lebanon; thus, this influx of refugees might have played a role in the increase of T2D observed in Lebanese males. It is possible that this population are now faced with higher levels of chronic stress themselves, whether due to limited resources, competition for work opportunities, or overpopulation in the areas the PHCs are situated in, further shedding light on the complex relationship between stress and the development of T2D.

Compared to national rates, the relatively lower rates of T2D in our study populations may be an indicator of poor screening resulting from limited access to healthcare. Poor screening among refugees has been reported in the past and could explain the lower prevalence of T2D among Syrian refugees compared to their national rates. ${ }^{32}$ This may also be the case among underprivileged Lebanese with limited access to healthcare. Future endeavors for improved diabetes screening and improved reporting may be necessary to evaluate the real prevalence of this disease among patients accessing these primary healthcare facilities. Future studies can also focus on other chronic illnesses that may be prevalent among these populations, such as mental illness and hypertension, both of which have their own associated consequences on patient health and both of which may be associated with increased rates of T2D.

\section{CONCLUSIONS}

The massive influx of Syrian refugees into Lebanon has further exhausted available healthcare resources at a time when the demand for PHCs continues to increase due to the economic crisis in Lebanon. Chronic diseases such as T2D have been shown to be associated with stress, and the rates of T2D have increased over time in the Lebanese and Syrian patient populations visiting five PHCs across Lebanon. These rates are still lower than national rates, which could indicate inadequate screening measures among these populations due to limited access to healthcare.

\section{ETHICS APPROVAL}

Ethical approval was obtained from the IRB of the University of Balamand.

\section{FUNDING}

Not applicable

\section{AUTHORSHIP CONTRIBUTIONS}

$\mathrm{PM}$ and RT drafted the initial version of the manuscript. PM, RT, PZ and GN analyzed the data. PZ, GN, RH and WA contributed to the revision of the manuscript. All authors reviewed the final draft for approval.

\section{COMPETING INTERESTS}

The authors completed the Unified Competing Interest form at http://www.icmje.org/disclosure-of-interest/ (available upon request from the corresponding author) and declare no conflicts of interest.

\section{CORRESPONDENCE TO:}

\section{Ghassan Nabbout, MD}

University of Balamand, Faculty of Medicine

Tripoli, Lebanon

ghassan.nabbout@fty.balamand.edu

Submitted: June 09, 2021 GMT, Accepted: June 10, 2021 GMT 
This is an open-access article distributed under the terms of the Creative Commons Attribution 4.0 International License (CCBY-4.0). View this license's legal deed at http://creativecommons.org/licenses/by/4.0 and legal code at http://creativecommons.org/licenses/by/4.0/legalcode for more information. 


\section{REFERENCES}

1. UNHCR. Syria Regional Refugee Response. UNHCR. Published August 31, 2020. Accessed April 20, 2020. h ttps://data2.unhcr.org/en/situations/syria/location/71

2. World Population Review. Lebanese Population 2019. World Population Review. Published 2019. Accessed December 2019. https://worldpopulationrevi ew.com/countries/lebanon-population

3. Onishi N. Lebanon Worries That Housing Will Make Syrian Refugees Stay. Published 2013. Accessed February 13, 2020. https://www.nytimes.com/2013/1 2/11/world/middleeast/lebanon-worries-that-housin g-will-make-syrian-refugees-stay.html?pagewante $\underline{\mathrm{d}=\mathrm{all}}$

4. UNHCR Lebanon. Shelter. Published 2019. Accessed January 22, 2020. https://www.unhcr.org/lb/shelter

5. Ammar W, Kdouh O, Hammoud R, et al. Health system resilience: Lebanon and the Syrian refugee crisis. Journal of global health.

2016;6(2):020704-020704. doi:10.7189/jogh.06.02070 4

6. Benage M, Greenough PG, Vinck P, Omeira N, Pham P. An assessment of antenatal care among Syrian refugees in Lebanon. Confl Health. 2015;9:8. do i:10.1186/s13031-015-0035-8

7. Central Admission of Statistics. Population Statistics. Published 2020. Accessed January 22, 2020. http://www.cas.gov.lb/index.php/demographic-and-s ocial-en/population-en

8. UNHCR. Bekaa (Zahle). Published 2020. Accessed January 22, 2020. https://www.unhcr.org/lb/bekaa-za hle

9. Lebanese Ministry of Public Health. National PHC Network. Published 2020. Accessed January 22, 2020. https://www.moph.gov.lb/en/Pages/0/8116/nationalphc-network

10. Ouosh C, Eloul L, Ajlani R. Mental health of refugees and displaced persons in Syria and surrounding countries: a systematic review. Intervention. 2013;11:276-294.

11. Obeid OA, Hachem DH, Ayoub JJ. Refeeding and metabolic syndromes: two sides of the same coin. Nutrition \& diabetes. 2014;4(6):e120. doi:10.1038/nut d.2014.21
12. Amara AH, Aljunid SM. Noncommunicable diseases among urban refugees and asylum-seekers in developing countries: a neglected health care need. Globalization and Health. 2014;10(1):24. doi:10.1186/1 744-8603-10-24

13. Bartoli F, Carrà G, Crocamo C, Carretta D, Clerici M. Metabolic syndrome in people suffering from posttraumatic stress disorder: a systematic review and meta-analysis. Metab Syndr Relat Disord. 2013;11(5):301-308. doi:10.1089/met.2013.0010

14. Novak M, Bjorck L, Giang KW, Heden-Stahl C, Wilhelmsen L, Rosengren A. Perceived stress and incidence of Type 2 diabetes: a 35-year follow-up study of middle-aged Swedish men. Diabet Med. 2013;30(1):e8-16. doi:10.1111/dme.12037

15. Yun K, Hebrank K, Graber LK, Sullivan MC, Chen I, Gupta J. High prevalence of chronic noncommunicable conditions among adult refugees: implications for practice and policy. J Community Health. 2012;37(5):1110-1118. doi:10.1007/s10900-01 2-9552-1

16. Lyles E, Doocy S. Syrian refugee and Affected Host Population Health Access Survey in Lebanon. UNHCR. Published online July 2015:129.

17. Doocy S, Lyles E, Roberton T, Akhu-Zaheya L, Oweis A, Burnham G. Prevalence and care-seeking for chronic diseases among Syrian refugees in Jordan. BMC Public Health. 2015;15(1):1097. doi:10.1186/s128 89-015-2429-3

18. Nations Online Project. Political Map of Lebanon. Published online 2019.

19. Ahmadieh H, Itani H, Itani S, et al. Diabetes and depression in Lebanon and association with glycemic control: a cross-sectional study. Diabetes, metabolic syndrome and obesity: targets and therapy. 2018;11:717-728. doi:10.2147/DMSO.S179153

20. Trading Economics. Lebanon - Diabetes Prevalence (\% Of Population Ages 20 To 79). Published 2021. Accessed 2020. https://tradingecono mics.com/lebanon/diabetes-prevalence-percent-of-p opulation-ages-20-to-79-wb-data.html

21. Albache N, Al Ali R, Rastam S, Fouad FM, Mzayek F, Maziak W. Epidemiology of Type 2 diabetes mellitus in Aleppo, Syria. J Diabetes. 2010;2(2):85-91. doi:10.1111/j.1753-0407.2009.00063.x 
22. Al Ali R, Mzayek F, Rastam S, et al. Forecasting future prevalence of type 2 diabetes mellitus in Syria. BMC Public Health. 2013;13(1):507. doi:10.1186/147 1-2458-13-507

23. Montero Kuscevic CM, Radmard H. Syrian refugees in Lebanon: a spatial study. Applied Economics Letters. 2020;27(5):417-421. doi:10.1080/13 504851.2019.1623862

24. World Bank Group. The Mobility of Displaced Syrians: An Economic and Social Analysis. Published 2019. Accessed 2019. https://www.worldbank.org/en/c ountry/syria/publication/the-mobility-of-displaced-s yrians-an-economic-and-social-analysis

25. Doocy S, Lyles E, Fahed Z, Mkanna A, Kontunen K, Burnham G. Characteristics of Syrian and Lebanese Diabetes and Hypertension Patients in Lebanon. The Open Hypertension Journal. 2018;10. doi:10.2174/1876 526201810010060

26. Bizri AR, Dada BA, Haschicho MH. Defamed relations: host community and refugees. Lancet. 2019;393(10182):1697-1698. doi:10.1016/s0140-673 6(18)33181-7

27. Truppa C, Leresche E, Fuller AF, et al. Utilization of primary health care services among Syrian refugee and Lebanese women targeted by the ICRC program in Lebanon: a cross-sectional study. Conflict and Health. 2019;13(1):7. doi:10.1186/s13031-019-0190-4
28. Kyrou I, Tsigos C. Stress hormones: physiological stress and regulation of metabolism. Current Opinion in Pharmacology. 2009;9(6):787-793. doi:10.1016/j.cop h.2009.08.007

29. Mooy JM, de Vries H, Grootenhuis PA, Bouter LM, Heine RJ. Major stressful life events in relation to prevalence of undetected type 2 diabetes: the Hoorn Study. Diabetes Care. 2000;23(2):197-201. doi:10.233 7/diacare.23.2.197

30. Chrousos GP. Stress and disorders of the stress system. Nat Rev Endocrinol. 2009;5(7):374-381. doi:1 $\underline{0.1038 / \text { nrendo.2009.106 }}$

31. Connolly V, Unwin N, Sherriff P, Bilous R, Kelly W. Diabetes prevalence and socioeconomic status: a population based study showing increased prevalence of type 2 diabetes mellitus in deprived areas. Journal of Epidemiology and Community Health. 2000;54(3):173. doi:10.1136/jech.54.3.173

32. Gaziza H, Shamsi H. Prevalence, management and control of diabetes mellitus among Syrian refugees in Duhok governorate , Kurdistan Region of Iraq: a cross sectional study in the camp of Domiz 1. Published online 2017. 